\title{
KASCADE-Grande energy spectrum of cosmic rays interpreted with post-LHC hadronic interaction models
}

M. Bertaina*3, W.D. Apel ${ }^{1}$, J.C. Arteaga-Velázquez ${ }^{2}$, K. Bekk ${ }^{1}$, J. Blümer ${ }^{1,4}$, H. Bozdog ${ }^{1}$, I.M. Brancus ${ }^{5}$, E. Cantoni ${ }^{3,6}$, A. Chiavassa $^{3}$, F. Cossavella ${ }^{4}$, K. Daumiller ${ }^{1}$, V. de Souza ${ }^{7}$, F. Di Pierro ${ }^{3}$, P. Doll ${ }^{1}$, R. Engel ${ }^{1}$, D. Fuhrmann ${ }^{8}$, A. Gherghel-Lascu ${ }^{5}$, H.J. Gils ${ }^{1}$, R. Glasstetter ${ }^{8}$, C. Grupen ${ }^{9}$, A. Haungs ${ }^{1}$, D. Heck ${ }^{1}$, J.R. Hörandel ${ }^{10}$, D. Huber ${ }^{4}$, T. Huege ${ }^{1}$, K.-H. Kampert ${ }^{8}$, D. Kang ${ }^{4}$, H.O. Klages ${ }^{1}$, K. Link ${ }^{4}$, P. Łuczak ${ }^{11}$, H.J. Mathes ${ }^{1}$, H.J. Mayer ${ }^{1}$, J. Milke ${ }^{1}$, B. Mitrica ${ }^{5}$, C. Morello ${ }^{6}$, J. Oehlschläger ${ }^{1}$, S. Ostapchenko ${ }^{12}$, N. Palmieri ${ }^{4}$, T. Pierog ${ }^{1}$, H. Rebel ${ }^{1}$, M. Roth ${ }^{1}$, H. Schieler ${ }^{1}$, S. Schoo ${ }^{1}$, F.G. Schröder ${ }^{1}$, O. Sima ${ }^{13}$, G. Toma ${ }^{5}$, G.C. Trinchero ${ }^{6}$, H. Ulrich ${ }^{1}$, A. Weindl ${ }^{1}$, J. Wochele ${ }^{1}$, J. Zabierowski ${ }^{11}$ - KASCADE-Grande Collaboration

${ }^{1}$ Institut für Kernphysik, KIT - Karlsruhe Institute of Technology, Germany

${ }^{2}$ Universidad Michoacana, Inst. Física y Matemáticas, Morelia, Mexico

${ }^{3}$ Dipartimento di Fisica, Università degli Studi di Torino, Italy

${ }^{4}$ Institut für Experimentelle Kernphysik, KIT - Karlsruhe Institute of Technology, Germany

${ }^{5}$ Horia Hulubei National Institute of Physics and Nuclear Engineering, Bucharest, Romania

${ }^{6}$ Osservatorio Astrofisico di Torino, INAF Torino, Italy

${ }^{7}$ Universidade São Paulo, Instituto de Física de São Carlos, Brasil

${ }^{8}$ Fachbereich Physik, Universität Wuppertal, Germany

${ }^{9}$ Department of Physics, Siegen University, Germany

${ }^{10}$ Dept. of Astrophysics, Radboud University Nijmegen, The Netherlands

${ }^{11}$ National Centre for Nuclear Research, Department of Astrophysics, Lodz, Poland

${ }^{12}$ Frankfurt Institute for Advanced Studies (FIAS), Frankfurt am Main, Germany

${ }^{13}$ Department of Physics, University of Bucharest, Bucharest, Romania

E-mail: bertaina@to.infn.it

Previous results obtained by KASCADE-Grande using QGSjetII-02, EPOS1.99 and SIBYLL hadronic interaction models have shown that the energy spectrum of cosmic rays between $10^{16} \mathrm{eV}$ and $10^{18} \mathrm{eV}$ exhibits a significant hardening at approximately $2 \times 10^{16} \mathrm{eV}$, a slight but statistically significant steepening close to $10^{17} \mathrm{eV}$, i.e. the 'knee' caused by the heavy component of primary cosmic rays, and an 'ankle' like feature of the light component just above $10^{17} \mathrm{eV}$. In this paper, we report on results of similar analyses performed using the post-LHC versions of the models QGSJetII-04 and EPOS-LHC.

The 34th International Cosmic Ray Conference,

30 July- 6 August, 2015

The Hague, The Netherlands

\footnotetext{
* Speaker.
} 


\section{Introduction}

The recent findings of KASCADE-Grande $[1,2,3]$ indicate that there are some features in the all-particle energy spectrum and in the spectra of the mass-groups in the energy range $10^{16}$ $-10^{18} \mathrm{eV}$. The details of these findings rely on the results of simulations and the description of hadronic interactions for reconstructing the properties of the primary particle which differ in predictions. However, a cross-check of the results obtained with various pre-LHC interaction models showed that, qualitatively, similar features are present in the spectra, independently of the interaction model used to interpret the data $[4,5,6]$.

In this paper, we present the results on the all-particle energy spectrum and mass-group separation of KASCADE-Grande data interpreted using the post-LHC versions of the EPOS [7] and QGSJetII [8] high-energy hadronic interaction models in the CORSIKA framework [9], namely EPOS-LHC and QGSJetII-04. Such results are compared to those obtained using the pre-LHC versions of these models, EPOS 1.99 and QGSjetII-02, as well as those obtained using SIBYLL $2.1[10]$ hadronic interaction model (see $[4,5,6]$ for details). Moreover, the analysis presented in this paper is based on the full statistics accumulated by KASCADE-Grande during its operational time between 2002 and 2013.

The technique to infer the energy spectrum and mass separation is the same as in the QGSjetII02 analyses [1, 2, 3] and it is described in detail in [11]. In the following, the names are abbreviated as SIBYLL, EPOS (for EPOS 1.99), EPOS-LHC, QGS2v4 (for QGSJetII-04) and QGSjet (for QGSJetII-02), respectively. In all cases, FLUKA [12] is used to describe the low-energy interactions in air-shower development.

\section{The technique}

The technique employed to derive the all-particle energy spectrum and the abundance of 'light' and 'heavy' primaries is based on the correlation between the number of charged particles $\left(\mathrm{N}_{c h}\right)$ with energy $\mathrm{E}>3 \mathrm{MeV}$, and muons $\left(\mathrm{N}_{\mu}\right)$ with kinetic energy $\mathrm{E}>230 \mathrm{MeV}$ on an event-by-event basis. Grande stations provide the core position and angle-of-incidence, as well as the total number of charged particles in the shower at observation level. The values are calculated by means of a maximum likelihood procedure comparing the measured number of particles with the one expected from a modified NKG lateral distribution function. The total number of muons is calculated using the core position determined by the Grande array and the muon densities measured by the KASCADE muon array detectors. Also in this case the total number of muons $N_{\mu}$ in the shower disk is derived from a maximum likelihood estimation where the lateral distribution function is based on the one proposed by [13]. The reconstruction procedures and accuracies of KASCADE-Grande observables are described in detail in [14] and related references therein.

Sets of simulated events were produced in the energy range from $10^{15} \mathrm{eV}$ to $3 \times 10^{18} \mathrm{eV}$ with high statistics and for five elements: $\mathrm{H}, \mathrm{He}, \mathrm{C}, \mathrm{Si}$ and $\mathrm{Fe}$, as representatives for different mass groups.

For the reconstruction of experimental events and simulated data, we restricted ourselves to events with zenith angles less than $40^{\circ}$. Additionally, only air showers with cores located in a central area of the KASCADE-Grande array were selected $\left(\sim 0.15 \mathrm{~km}^{2}\right)$. With these cuts on the 
fiducial area, border effects are discarded and possible under- and overestimations of the muon number for events close to and far away from the center of the KASCADE array are reduced. All of these cuts were applied also to the Monte Carlo simulations to study their effects. Full efficiency for triggering and reconstruction of air-showers is reached at a primary energy of $\approx$ $10^{16} \mathrm{eV}$. The analysis presented here is based on a total time of 1753 days between 2002 and 2013 with the requirement that KASCADE-Grande operated with good data quality. The cuts on the sensitive central area and zenith angle correspond to a total acceptance of $A=0.1976 \mathrm{~km}^{2}$. sr, and an exposure of $N=0.949 \mathrm{~km}^{2} \cdot \mathrm{sr} \cdot$ year, respectively.

Based on Monte-Carlo simulations a formula is obtained to calculate the primary energy per individual shower on the basis of the reconstructed $N_{c h}$ and $N_{\mu}$. The formula takes into account the mass sensitivity in order to minimize the composition dependence in the energy assignment, and at the same time, provides an event-by-event separation between 'light' and 'heavy' candidates. The formula is defined for 5 different zenith angle intervals independently, to take into account the shower attenuation in the atmosphere. Data are combined only at the very last stage to obtain a unique spectrum. The energy assignment is defined as $E=f\left(N_{c h}, k\right)$ (see equation 2.1), where $\mathrm{N}_{c h}$ is the number of charged particles and the parameter $k$ is defined through the ratio of the numbers of the $\mathrm{N}_{c h}$ and $\mathrm{N}_{\mu}$ components: $k=g\left(N_{c h}, N_{\mu}\right)$ (see equation 2.2). The main aim of the $k$ variable is to take into account the average differences in the $\mathrm{N}_{c h} / \mathrm{N}_{\mu}$ ratio among different primaries with similar $\mathrm{N}_{c h}$ and the shower to shower fluctuations for events of the same primary mass :

$$
\begin{gathered}
\log _{10} E=\left[a_{H}+\left(a_{F e}-a_{H}\right) \cdot k\right] \cdot \log _{10} N_{c h}+b_{H}+\left(b_{F e}-b_{H}\right) \cdot k \\
k=\frac{\log _{10}\left(N_{c h} / N_{\mu}\right)-\log _{10}\left(N_{c h} / N_{\mu}\right)_{H}}{\log _{10}\left(N_{c h} / N_{\mu}\right)_{F e}-\log _{10}\left(N_{c h} / N_{\mu}\right)_{H}} \\
\log _{10}\left(N_{c h} / N_{\mu}\right)_{H, F e}=c_{H, F e} \cdot \log _{10} N_{c h}+d_{H, F e} \cdot d_{H, F e} .
\end{gathered}
$$

The $k$ parameter is, by definition of eq. (2.2), a number centered around 0 for $\mathrm{H}$ initiated showers and 1 for Fe ones if expressed as a function of $N_{c h}$ for Monte Carlo events. It is expected that the average values of the $k$ paramenter for the experimental data lie between the $\mathrm{H}$ and $\mathrm{Fe}$ limits. In case this is not verified it would be a hint of some deficit of the model to describe the experimental data. Naturally, as the calibration functions differ from model to model, the same experimental event might give different values of $k$ when different calibration functions are used.

Simulated events using a mixture of all primaries have been divided in bins of true energy and the distributions of the relative differences between reconstructed and true energies have been created. The RMS of such distributions (energy resolution) is $\sim 26 \%$ at the energy threshold and decreases with energy, due to the lower fluctuations of the shower development and reconstruction uncertainties, becoming $<20 \%$ at the highest energies. The ratio of the reconstructed flux over the true one in each energy differs by less than $10 \%$ from unity. This results applies also for pure light $(50 \% \mathrm{H}-50 \% \mathrm{He})$ or pure heavy $(50 \% \mathrm{Si}-50 \% \mathrm{Fe})$ compositions. A similar behavior exists for all hadronic interaction models.

Assuming QGSjet as the reference model for a fixed energy, EPOS-LHC, QGS2v4 and EPOS show a higher muon content, while SIBYLL simulated events show less amount of electrons and muons. As a consequence, when interpreting the same experimental event, EPOS-LHC, QGS2v4 and EPOS are expected to assign a lower energy than QGSjet, while SIBYLL a higher one. This is 


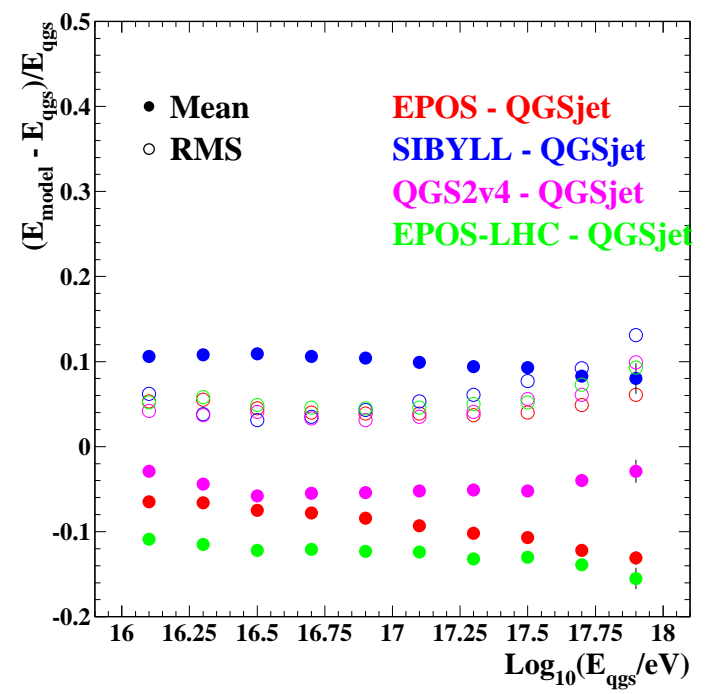

Figure 1: Difference between the energy reconstructed by EPOS-LHC (filled green dots), QGS2v4 (filled pink dots), SIBYLL (filled blue dots), EPOS (filled red dots) on experimental data compared to QGSjet as a function of the energy reconstructed by QGSjet. The open dots refer to the width of the distributions in each energy bin.

confirmed by Fig. 1. In that figure, the average relative difference between the energy reconstructed on experimental data by EPOS-LHC, QGS2v4, EPOS and SIBYLL compared to QGSjet on an event-by-event basis, for different energy bins is shown. The post-LHC models assign an average of 5\% (QGS2v4) - 12\% (EPOS-LHC) lower energy than QGSjet. The pre-LHC models EPOS and SIBYLL give a $10 \%$ lower and higher energy, respectively.

\section{The energy spectrum}

Applying the energy calibration functions obtained by each model to the measured data, the all-particle energy spectra for the five zenith angle bins are obtained for QGSjet, EPOS-LHC, QGS2v4, SIBYLL and EPOS. For all the models an unfolding procedure has been applied as well. Different sources of uncertainty affect the all-particle energy spectrum. A detailed description is reported in [2]. They take into account: a) the angular dependence of the parameters appearing in the energy calibration functions of the different angular ranges. b) The possible bias introduced in the energy spectrum by different primary compositions. c) The spectral slope of Monte Carlo used in the simulations. d) The reconstruction quality of $N_{c h}$ and $N_{\mu}$. The total systematic uncertainty is $\sim 20 \%$ at the threshold $\left(\mathrm{E}=10^{16} \mathrm{eV}\right)$ and $\sim 30 \%$ at the highest energies $\left(\mathrm{E}=10^{18} \mathrm{eV}\right)$ almost independently from the interaction model used to interpret the data. The final all-particle spectrum of KASCADE-Grande is obtained (see Fig. 2) by combining the spectra for the individual angular ranges. Only those events are taken into account, for which the reconstructed energy is above the energy threshold for the angular bin of interest. In general the shape of the energy spectrum is very similar for the five models, however, a shift in flux is clearly observed which amounts to $\sim 25 \%$ increase in case of SIBYLL and $\sim 15 \%$ decrease in case of EPOS-LHC. This is the consequence 


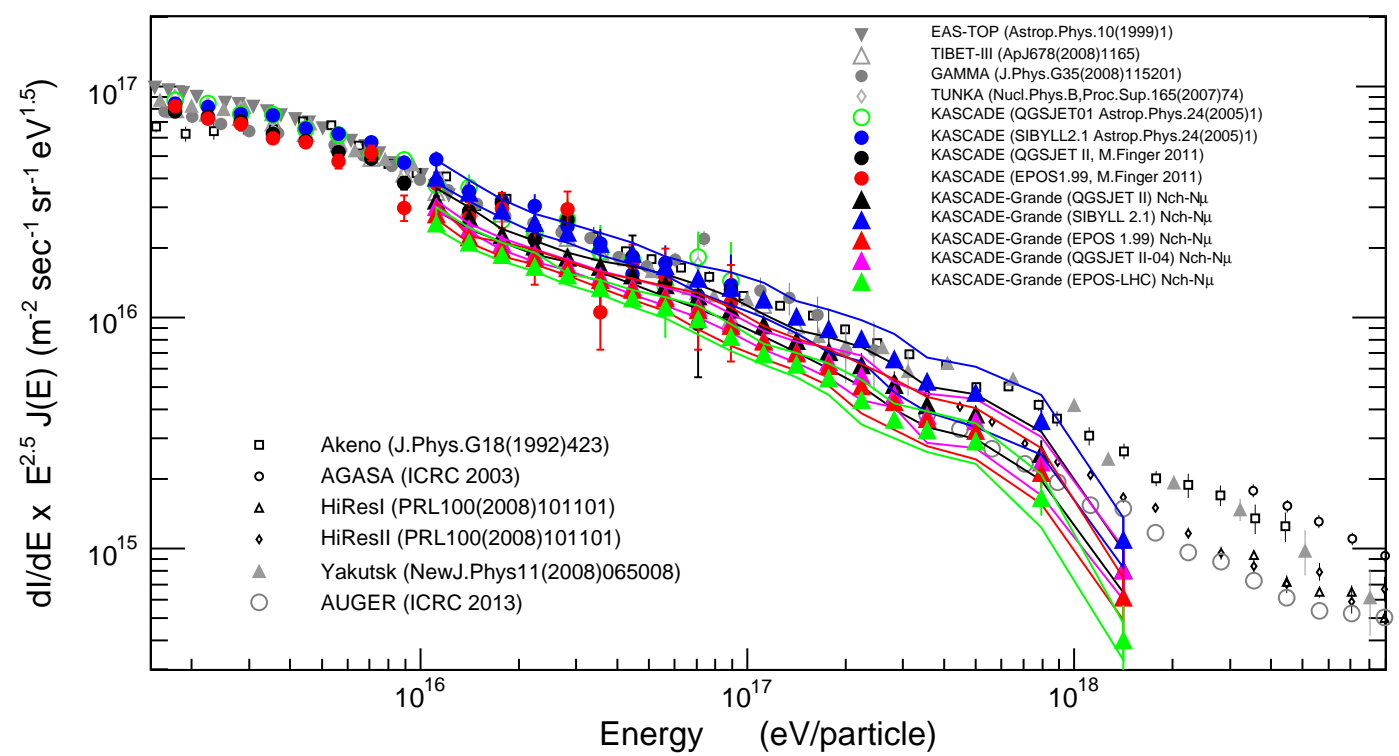

Figure 2: Comparison of the all-particle energy spectrum obtained with KASCADE-Grande data based on SIBYLL (blue), QGSJet (black), QGS2v4 (pink), EPOS (red) and EPOS-LHC (green) models to results of other experiments. The band denotes the systematic uncertainties in the flux estimation.

of the energy shift assigned on an event-by-event basis previously discussed. This result gives an estimation of the systematic uncertainty on the experimental flux due to the hadronic interaction model used to interpret the data, and it is essentially independent of the technique used to derive the flux, namely averaging the fluxes obtained in different angular bins. In general the post-LHC models tend to decrease the flux compared to the original QGSjet energy spectrum by $5 \%-15 \%$. The shift in the assigned energy to the data is also visible in the hardening around $\sim 2 \times 10^{16} \mathrm{eV}$ and in the steepening around $10^{17} \mathrm{eV}$ which look shifted among the models in general agreement with the energy shift. This result indicates that the features seen in the spectrum are not an artefact of the hadronic interaction model used to interpret the data but they are in the measured data. In the overlapping region, KASCADE-Grande data are compatible inside the systematic uncertainties with KASCADE data interpreted with the same model.

\section{The separation into mass groups}

The mass-group separation is performed subdividing the measured data in two samples: the electron-poor and electron-rich samples based on the $k$ parameter - see equation 2.2. A detailed explanation of the procedure is reported in $[1,3]$. The electron-poor and electron-rich samples, defined in this way, indicate the 'heavy' and 'light' mass-groups, respectively. The analysis is conducted independently for each hadronic interaction model. In each energy bin the average value of $k$ for pure $\mathrm{H}, \mathrm{He}, \mathrm{C}, \mathrm{Si}$ and $\mathrm{Fe}$ simulated compositions is evaluated. These values are very similar among models by construction (see equation 2.2). In fact $\mathrm{H}$ showers will lead to average 

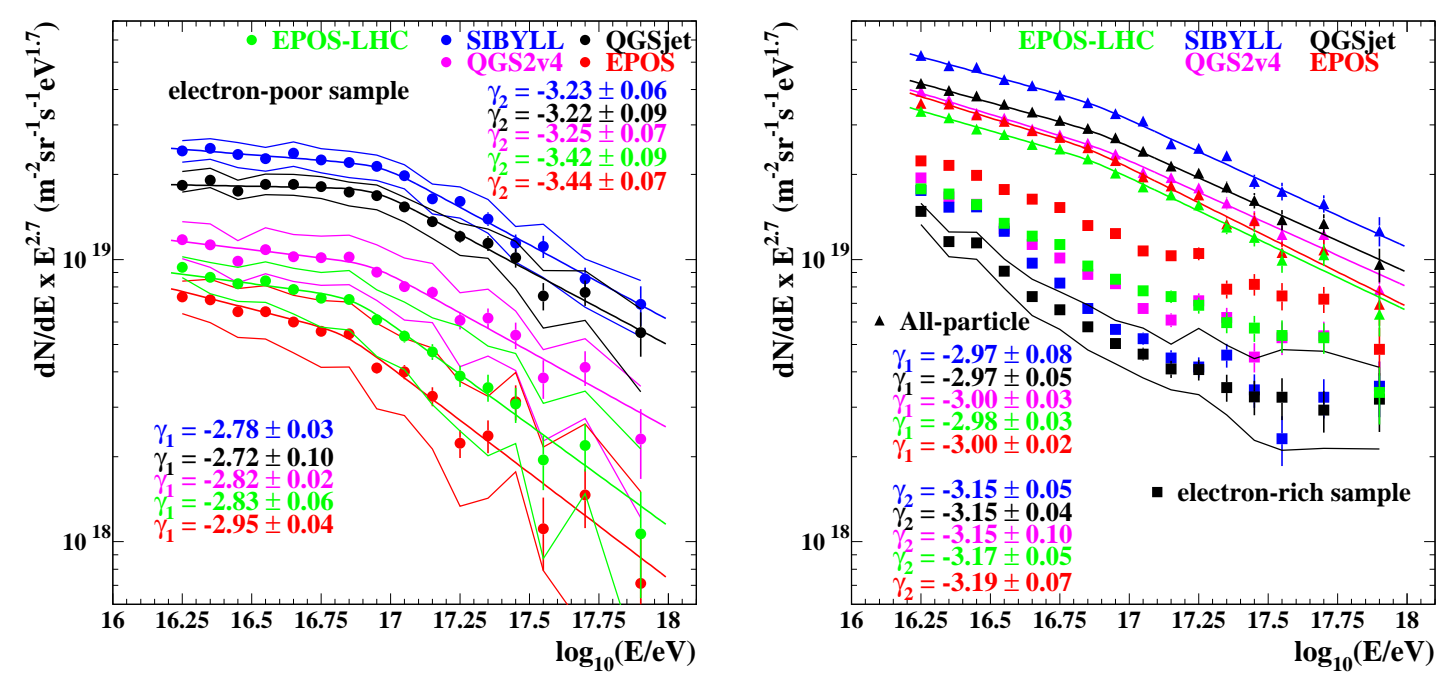

Figure 3: Reconstructed energy spectra of the heavy (left plot) and light components together with the allparticle spectrum (right plot) for the five hadronic interaction models. The error bars show the statistical uncertainties; the bands assign systematic ones due to the selection of subsamples. For the light component only the systematic uncertainties of QGSjet have been indicated, anyhow they are similar in all models. Fits on the spectra and resulting slopes are also indicated.

$k$ values close to 0 and $\mathrm{Fe}$ showers close to 1 . Two lines are used to separate events into heavy $\left(k(E)>k_{h}(E)\right)$ and light mass groups $\left(k(E)<k_{l}(E)\right.$ ), where the separation line of the heavy massgroup is defined by fitting the $k_{h}(E)=\left(k_{S i}(E)+k_{C}(E)\right) / 2$ points which are obtained by averaging the values of $k$ for $\mathrm{Si}$ and $\mathrm{C}$ components of the simulated events, and the light mass-group is defined by fitting the $k_{l}(E)=\left(k_{C}(E)+k_{H e}(E)\right) / 2$ points which are obtained by averaging the values of $k$ for $\mathrm{C}$ and $\mathrm{He}$ components of the simulated events. Naturally, the absolute abundances of the experimental data in the two samples depend on the value of the cut lines. However, the evolution of the abundances as a function of energy will be retained by this approach, as the lines are defined through a fit to the $k$ values. The assignment to the heavy or light mass groups is performed on an event-by-event basis. Due to the different $N_{c h} / N_{\mu}$ ratio among models for the same $k$ value, the same experimental event might be assigned to the same group, to none of them or even to a different group depending on the model used. As a consequence, the abundances of the so defined heavy and light groups will vary among models. The abundances of the heavy (left plot) and of the light (right plot) according to the different hadronic interaction models used to interpret the data are shown in Fig. 3. With such a selection cut the reconstructed spectrum of the heavy primary sample shows a distinct knee-like feature around $10^{17} \mathrm{eV}$ for all hadronic interaction models. Applying a fit of two power laws to the spectrum interconnected by a smooth knee in the entire energy range 16.2 $<\log _{10}(E / e V)<18.0$ results in a statistical significance that the entire spectrum cannot be fitted with a single power-law. These results are summarized in tab.1. The spectrum of the electron-rich component is much steeper with a possible hardening at the highest energies for all models. Details are discussed in $[3,15]$. 


\begin{tabular}{|lccccc|}
\hline Model & EPOS & EPOS-LHC & QGS2v4 & QGSjet & SIBYLL \\
\hline All-particle & & & & \\
$\gamma_{1}$ & $-3.00 \pm 0.02$ & $-2.98 \pm 0.03$ & $-3.00 \pm 0.03$ & $-2.97 \pm 0.05$ & $-2.97 \pm 0.08$ \\
$\gamma_{2}$ & $-3.19 \pm 0.07$ & $-3.17 \pm 0.05$ & $-3.15 \pm 0.10$ & $-3.15 \pm 0.05$ & $-3.15 \pm 0.05$ \\
$\log (E / e V)$ & $16.86 \pm 0.10$ & $16.87 \pm 0.12$ & $16.91 \pm 0.24$ & $16.88 \pm 0.16$ & $16.87 \pm 0.16$ \\
signif. $(\sigma)$ & 4.4 & 3.0 & 2.8 & 7.4 & 2.7 \\
\hline Heavy component & \multicolumn{5}{c}{} \\
$\gamma_{1}$ & $-2.95 \pm 0.04$ & $-2.83 \pm 0.06$ & $-2.82 \pm 0.02$ & $-2.72 \pm 0.10$ & $-2.78 \pm 0.03$ \\
$\gamma_{2}(\mathrm{~F} / \mathrm{eV})$ & $-3.44 \pm 0.07$ & $-3.42 \pm 0.09$ & $-3.25 \pm 0.07$ & $-3.22 \pm 0.09$ & $-3.23 \pm 0.06$ \\
$\log (E .83 \pm 0.05$ & $16.87 \pm 0.09$ & $16.93 \pm 0.06$ & $16.94 \pm 0.09$ & $16.97 \pm 0.05$ \\
$\operatorname{signif.}(\sigma)$ & 3.0 & 11.0 & 3.7 & 9.7 & 11.6 \\
\hline
\end{tabular}

Table 1: Slope of the different spectra and break positions obtained with the five different hadronic interaction models, by applying the $k$ parameter analysis in order to extract the spectrum of the heavy component.

\section{Conclusions}

The energy spectrum and separation into mass-groups have been obtained for the post-LHC models EPOS-LHC and QGS2v4. The results have been compared to those obtained using SIBYLL, EPOS and QGSjet hadronic interaction models using the same approach. The results confirm qualitatively the previous findings. The all-particle spectrum in the range $10^{16}-10^{18} \mathrm{eV}$ is found to exhibit some smaller structures: In particular, a hardening of the spectrum at $\sim 2 \times 10^{16} \mathrm{eV}$, and a small break-off at $\sim 8 \times 10^{16} \mathrm{eV}$. The energy position of such features slightly depends on the energy assigned by the interaction model to the event. In general is at lower energies for EPOS-LHC and QGS2v4 compared to QGSjet. The separation into mass groups performed via the $k$ parameter reveals that the knee-like feature around $10^{17} \mathrm{eV}$ in the all-particle spectrum is associated with a break in the heavy component. The abundance of the heavy component varies significantly among models, however EPOS-LHC and QGS2v4 show similar behavior. In this sense the interpretation of which mass group is responsible for this break strongly depends on the hadronic interaction model employed to interpret the data. The spectrum of the electron-rich component is much steeper with a tendency for hardening above $10^{17} \mathrm{eV}$ for all models.

Acknowledgment: The authors would like to thank the members of the engineering and technical staff of the KASCADE-Grande collaboration, who contributed to the success of the experiment. The KASCADE-Grande experiment is supported in Germany by the BMBF and by the 'Helmholtz Alliance for Astroparticle Physics - HAP' funded by the Initiative and Networking Fund of the Helmholtz Association, by the MIUR and INAF of Italy, the Polish Ministry of Science and Higher Education, and the Romanian Authority for Scientific Research UEFISCDI (PNII-IDEI grants 271/2011 and 17/2011). J.C.A.V. acknowledges the partial support of CONACyT.

\section{References}

[1] W.-D. Apel et al. - KASCADE-Grande Coll., Phys. Rev. Lett. 107 (2011) 171104.

[2] W.-D. Apel et al. - KASCADE-Grande Coll., Astrop. Phys. 36 (2012) 183.

[3] W.-D. Apel et al. - KASCADE-Grande Coll., Phys. Rev. D 87 (2013) 081101.

[4] W.-D. Apel et al. - KASCADE-Grande Coll., Advan. in Space Res. 53/10 (2013) 1456.

[5] M. Bertaina et al. - KASCADE-Grande Coll., Proc. 33 ${ }^{\text {rd }}$ ICRC (2013) \#0196.

[6] M. Bertaina et al. - KASCADE-Grande Coll., Nucl. Phys. B (Proc. Suppl.) 256-257 (2014) 149. 
[7] K. Werner et al., Phys. Rev. C 74 (2006) 044902.

[8] S. Ostapchenko, Phys. Rev. D 74 (2006) 014026.

[9] D. Heck et al., FZKA6019 (1998).

[10] J. Engel et al., Phys. Rev. D 46 (1992) 5013.

[11] M. Bertaina et al. - KASCADE-Grande Coll., Astroph. and Space Science Trans. 7 (2011) 229.

[12] G. Battistoni et al., AIP Conf. Proc. 896 (2007) 31.

[13] A.-A. Lagutin et al., Nucl. Phys. B (Proc. Suppl.) 97 (2001) 274.

[14] W.-D. Apel et al. - KASCADE-Grande Coll., Nucl. Instr. Meth. Phys. Res., Sect. A 620 (2010) 202.

[15] S. Schoo et al. - KASCADE-Grande Coll., Proc. $33^{r d}$ ICRC (2013) \#0527. 